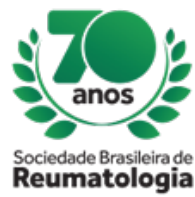

\title{
TAKAYASU ARTERY OF SLOW EVOLUTION
}

GILBERTO SCANAGATTA (Universidade Federal de Ciências da Saúde de Porto Alegre , PORTO ALEGRE, RS, Brasil), TATIANA FREITAS TOURINHO (Universidade Federal de Ciências da Saúde de Porto Alegre, PORTO ALEGRE, RS, Brasil), MARIA LUCIA LEMOS LOPES (Universidade Federal de Ciências da Saúde de Porto Alegre, PORTO ALEGRE, RS, Brasil), MARIA ODETE ESTEVES HILARIO (Universidade Federal de Ciências da Saúde de Porto Alegre, PORTO ALEGRE, RS, Brasil), RAISSA VELASQUES DE FIGUEIREDO (Universidade Federal de Ciências da Saúde de Porto Alegre, PORTO ALEGRE, RS, Brasil), RAFAEL CORADIN (Universidade Federal de Ciências da Saúde de Porto Alegre, PORTO ALEGRE, RS, Brasil), LUANA RIBEIRO CARLOS (Universidade Federal de Ciências da Saúde de Porto Alegre, PORTO ALEGRE, RS, Brasil), THIAGO WILLERS (Universidade Federal de Ciências da Saúde de Porto Alegre , PORTO ALEGRE, RS, Brasil)

\section{BACKGROUND}

The Takayasu's arteritis is a vasculitis of medium and large vessels that affects mainly the aorta and its main branches. It is more common in young women and tends to give symptoms such as blood pressure difference between the members, the absence of radial pulse among others.

In this report, we explain the case of a patient with Takayasu arteritis with oligosymptomatic evolution generating a major vessel obstruction.

\section{CASE REPORT}

Female patient, 57 years old, Caucasian, teacher. Consulted due to intermittent gluteal claudication. Associated with these symptoms, she also had dyspnea on minimal exertion. Patient does not know how long these symptoms have been. Upon physical examination, the patient had just a left carotid artery, and a right radial absent pulse. She had angiotomography of the abdomen and pelvis showing right renal artery with stenosis and atrophic kidney. Right iliac artery with total occlusion. Left common iliac artery with stenosis of $50-75 \%$.

In the return visit the patient brought carotid ecodoppler, which showed alterations determining diffuse changes of the right common carotid artery and carotid artery with reverse flow. Left, plaques throughout the artery not determining hemodynamic changes. The patient remained with complaints of lameness, worsening in the upper limbs, (mainly to the right).

The patient then returned to the consultation with angiotomography of the thorax, cervical and upper limbs showing brachiocephalic trunk with critical occlusion. Recirculation by collaterals coming out of the subclavian artery. Right common carotid artery with irregularities. The common carotid artery with the same irregularities, determining $50 \%$ of stenosis. Subclavian artery with luminal reduction of $70 \%$. Also had the following results: ANA 1: 160 homogeneous standard, Anti SSA, Anti SSB, Anti SM, Anticardiolipin IgM negative and absent lupus anticoagulant. It also presented a search for cryoglobulins, HIV, HVC and HBV negative .

At follow-up, the patient remained symptomatic, not tolerating the reduction of the corticoid. We then chose to start anti-TNF (infliximab). While the patient is awaiting medication, she has been making monthly pulses (already performed 2 cycles) with $1 \mathrm{~g}$ of cyclophosphamide, with symptomatic improvement and tolerating reduction of corticoid.

\section{CONCLUSION}

Takayasu's arteritis is a vasculitis of medium and large vessels that primarily affects young women, but can affect patients at any age. 
In this report we present a case of a 57 year old patient with an important involvement of aortic branches that remained oligosymptomatic for a long period, thus delaying the diagnosis and management of this vasculitis. 